\title{
Improving Performance of InGaN/GaN Light-Emitting Diodes and GaAs Solar Cells Using Luminescent Gold Nanoclusters
}

\author{
M. D. Yang, ${ }^{1,2}$ S. W. Wu, ${ }^{1}$ G. W. Shu, ${ }^{1}$ J. S. Wang, ${ }^{1}$ J. L. Shen, ${ }^{1}$ C. H. Wu, ${ }^{2}$ C. A. J. Lin, ${ }^{3}$ \\ W. H. Chang, ${ }^{3}$ T. Y. Lin, ${ }^{4}$ T. C. Lu, ${ }^{5}$ and H. C. Kuo ${ }^{5}$ \\ ${ }^{1}$ Department of Physics, Center for Nanotechnology, Chung Yuan Christian University (cycu), Chung-Li 32023, Taiwan \\ ${ }^{2}$ Institute of Nuclear Energy Research, P.O. Box 3-11, Lungtan 32546, Taiwan \\ ${ }^{3}$ Department of Biomedical Engineering, Chung Yuan Christian University, Chung-Li 32023, Taiwan \\ ${ }^{4}$ Institute of Optoelectronic Sciences, National Taiwan Ocean University, Keelung 20224, Taiwan \\ ${ }^{5}$ Department of Photonics and Institute of Electro-Optical Engineering, National Chiao-Tung University, Hsin-Chu 30010, Taiwan
}

Correspondence should be addressed to J. L. Shen, jlshen@cycu.edu.tw

Received 20 January 2009; Accepted 26 August 2009

Recommended by Ching Ping Wong

\begin{abstract}
We studied the optoelectronic properties of the InGaN/GaN multiple-quantum-well light emitting diodes (LEDs) and singlejunction GaAs solar cells by introducing the luminescent Au nanoclusters. The electroluminescence intensity for InGaN/GaN LEDs increases after incorporation of the luminescent Au nanoclusters. An increase of $15.4 \%$ in energy conversion efficiency is obtained for the GaAs solar cells in which the luminescent Au nanoclusters have been incorporated. We suggest that the increased light coupling due to radiative scattering from nanoclusters is responsible for improving the performance of the LEDs and solar cells.
\end{abstract}

Copyright ( 2009 M. D. Yang et al. This is an open access article distributed under the Creative Commons Attribution License, which permits unrestricted use, distribution, and reproduction in any medium, provided the original work is properly cited.

\section{Introduction}

The optical properties of metal nanoparticles are of great fundamental and technological interest due to the strong surface plasmon resonance in the visible region. Metal nanoparticles play an important role in mediating nonlinear processes such as surface enhanced Raman scattering, increasing the outcoupling of light from diodes, and enhancing the photovoltaic conversion efficiency of solar cells [17]. One explanation of the observed enhancement effects is the resonant light absorption in the metal nanoparticles, which may be caused by local plasmon excitation and interband transitions in the metal nanoparticles [8]. The use of enhancement effects for improving the performance of optoelectronic devices, such as light emitting diodes (LEDs), solar cells, and photodectors, is very promising. It has been reported that $\mathrm{Au}$ nanoparticles on $\mathrm{Si}$ pnjunction diodes increase the photogenerated current via surface plasmon resonance on nanoparticles [9]. An increase in electroluminescence (EL) and photocurrent has also been observed by introduction of Ag nanoparticles into siliconon-insulator LED $[4,5]$. Similarly, enhancement of the photovoltaic conversion efficiency of silicon solar cells and organic thin-film solar cells using $\mathrm{Ag}$ or Au nanoparticles has been demonstrated [6-8].

So far most of the metallic nanoparticles mentioned above have dimensions of particles about $5-100 \mathrm{~nm}$. As the metal particle size decreases further to contain several hundreds down to a few tens of atoms, some special properties begin to form because their electronic bands yield to quantum confinement effects and discrete electronic states develop. The metallic nanoclusters (small nanoparticles) display a strong luminescence from visible to near-infrared range, which may make them useful for applications in light emitting sources, chemical sensing, and biological labeling [10-13]. Recent studies have shown that similar enhancement in surface-enhancement Raman scattering and photoluminescence can be obtained by encapsulating metal nanoclusters [14, 15]. Also, the conversion efficiency of multiple-junction solar cells has been demonstrated to 


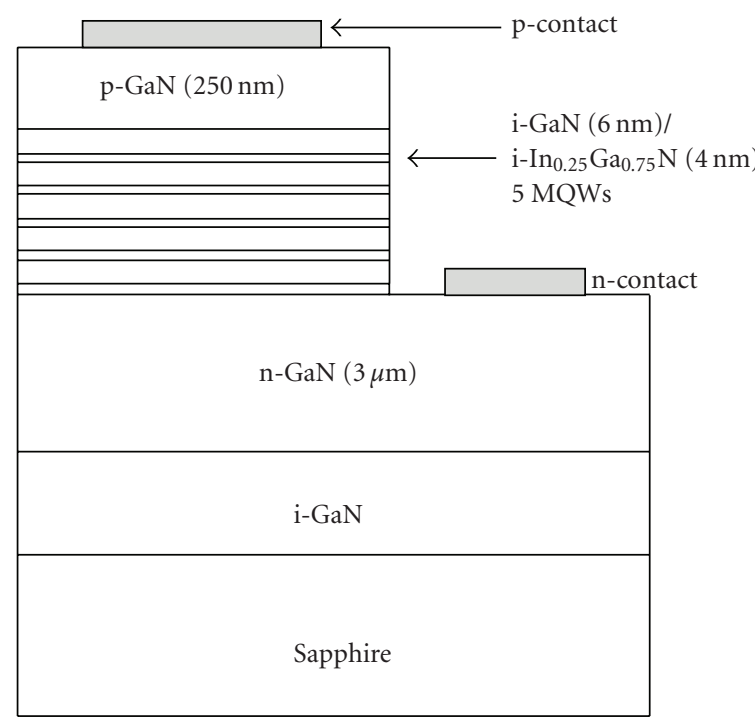

(a)

\begin{tabular}{|c|c|c|c|}
\hline $\mathrm{n}^{+}$ & GaAs & $0.25 \mu \mathrm{m}$ & $1 \times 10^{19} \mathrm{~cm}^{-3}$ (Si doped) \\
\hline $\mathrm{n}$ & $\mathrm{AI}_{0.5} \operatorname{In}_{0.5} \mathrm{P}$ & $0.06 \mu \mathrm{m}$ & $2 \times 10^{18} \mathrm{~cm}^{-3}($ Si doped $)$ \\
\hline $\mathrm{n}$ & GaAs & $0.1 \mu \mathrm{m}$ & $1 \times 10^{18} \mathrm{~cm}^{-3}($ Si doped $)$ \\
\hline $\mathrm{p}$ & GaAs & $3 \mu \mathrm{m}$ & $2 \times 10^{17} \mathrm{~cm}^{-3}(\mathrm{Zn}$ doped $)$ \\
\hline $\mathrm{p}$ & $\mathrm{In}_{0.5} \mathrm{Ga}_{0.5} \mathrm{P}$ & $0.1 \mu \mathrm{m}$ & $1 \times 10^{18} \mathrm{~cm}^{-3}(\mathrm{Zn}$ doped $)$ \\
\hline $\mathrm{p}$ & GaAs & $0.5 \mu \mathrm{m}$ & $3 \times 10^{18} \mathrm{~cm}^{-3}(\mathrm{Zn}$ doped $)$ \\
\hline $\mathrm{p}$ & $\mathrm{Ge}$ & $150 \mu \mathrm{m}$ & \\
\hline
\end{tabular}

(b)

FIGURE 1: Schematic cross-section of (a) the InGaN/GaN multiple-quantum-well LEDs and (b) InGaP/InGaAs/Ge solar cells.

increase by using $\mathrm{Au}$ nanoclusters [16]. In this paper, we study the influence of the luminescent Au nanoclusters on photovoltaic current-voltage $(I-V)$ characteristics and EL of the InGaN/GaN multiple-quantum-well (MQW) LEDs and single-junction GaAs solar cells, respectively. We obtain increases in EL intensity of InGaN/GaN LEDs by two times and in conversion efficiency of the GaAs solar cells by $15.4 \%$.

\section{Experiment}

The InGaN/GaN MQW LEDs employed in this study were grown on c-plane (0001) sapphire substrates with metal organic chemical vapor deposition. A nucleation $\mathrm{GaN}$ layer was first grown at the substrate, followed by a $3 \mu$-m thick $\mathrm{n}$-type GaN. The active layers consist of 5 periods of MQWs with 4-nm-thick InGaN wells and 6-nm-thick GaN barriers. Finally, the MQWs were capped with a 250-nm-thick p-GaN layer. The GaAs solar cells were grown by MOCVD on ptype $\mathrm{Ge}$ substrates. The GaAs and $\mathrm{InGaP}$ buffer layers were first grown at the substrate, followed by a $3 \mu \mathrm{m}$ thick ptype GaAs base layer, $100 \mathrm{~nm}$ n-type emitter layer, $60 \mathrm{~nm}$ AlInP window layer, and $250 \mathrm{~nm} \mathrm{GaAs} \mathrm{contact} \mathrm{layer.} \mathrm{The}$ schematics of the InGaN/GaN MQW LED and GaAs solar cell are shown in Figures 1(a) and 1(b), respectively. The luminescent $\mathrm{Au}$ nanoclusters investigated were prepared according to a modified Peng reaction [17]. In brief, the gold precursor solution was prepared by dissolving $\mathrm{AuCl}_{3}$ in the Didodecyldimethylammonium bromide (DDAB) solution. Decanoic acid was then combined with (Tetrabutylammonium borohydride) (TBAB) in toluence, followed by the gold precursor solution. The reduction of $\mathrm{Au}$ ions was realized quickly under vigorous stirring, leading to the formation of a dark-red Au colloid. The Au nanoclusters were further fragmented by adding the gold precursor solution and dodecanethiol (DDT) in toluene, leading to formation

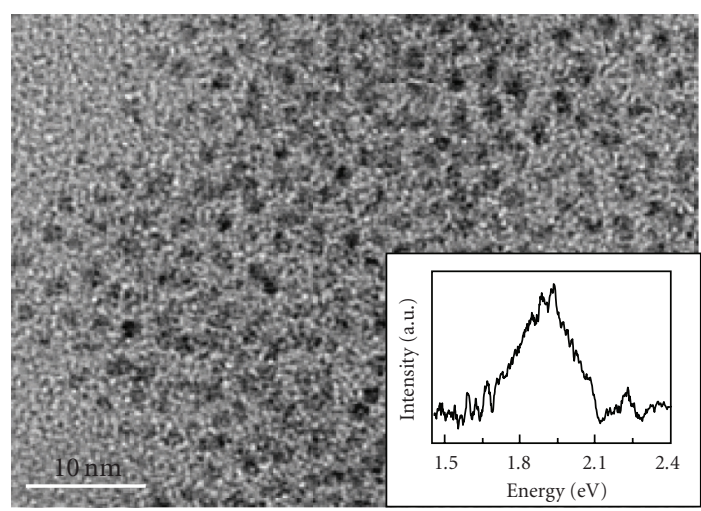

FIGURE 2: High-resolution transmission electron microscopy (HRTEM) image for the luminescent Au nanoclusters. The inset displays photoluminescence of the luminescent Au nanoclusters at room temperature.

of the luminescent $\mathrm{Au}$ nanoclusters. The luminescent $\mathrm{Au}$ nanoclusters were deposited onto the LED and solar-cell surfaces by drop casting a solution of nanoclusters, followed by drying at room temperature. In the EL measurements, the output EL intensity from the top side was collected and dispersed by a $0.75 \mathrm{~m}$ spectrometer and detected with a photomultiplier tube (PMT). $I-V$ characteristics of the solar cells were measured with a source meter (Keithley-2400) under simulated AM1.5 irradiation $\left(100 \mathrm{~mW} / \mathrm{cm}^{2}\right)$ from a solar simulator (WACOM, WXS-130S-L2+).

\section{Resutls and Discussion}

Figure 2 shows the high-resolution transmission electron microscopy (HRTEM) micrograph of the luminescent $\mathrm{Au}$ 
nanoclusters deposited on an electron microscope grid from colloidal solution. A uniform distribution of well-dispersed nanoclusters is seen. The statistics of the size distribution as determined from the previous and other micrographs obtained from different regions indicate an average size of $1.9 \pm 0.2 \mathrm{~nm}$. The inset of Figure 2 displays the roomtemperature photoluminescence spectrum from the luminescent $\mathrm{Au}$ nanoclusters with the $350 \mathrm{~nm}$ Xe-lamp excitation. A red luminescence between 1.7 and $2.1 \mathrm{eV}$ is observed with a prominent peak at $1.9 \mathrm{eV}$. The luminescence from the metal nanoclusters could be viewed as a radiative recombination from an excited-state highest occupied molecular orbital (HOMO) lowest unoccupied molecular orbital (LUMO) transition or the Fermi level electrons to occupied d-band holes $[18,19]$.

Figure 3 shows the $I-V$ characteristics of the LEDs before (close triangles) and after (open squares) deposition of the luminescent $\mathrm{Au}$ nanoclusters. Basically, the forward and revised $I-V$ characteristics of the two curves are almost identical. Nearly zero current in the reverse-biased voltage range reveals that the leakage currents in our LEDs are low. The threshold voltages of the two $I-V$ curves are similar; whereas the slope of the $I-V$ curve is steeper for the LEDs after introduction of the luminescent Au nanoclusters. The increase of slope in the $I$ - $V$ curve corresponds to a reduction in the series resistance, which is one of the factors that can determine the quality of the LED performance. To extract the series resistance of the LED, the slope of the $I-V$ curves was fitted by the diode equation

$$
I=I_{0}\left(\exp \left(\frac{q\left(V-R_{S}\right)}{n k T}\right)\right)
$$

where $n$ is the ideality factor, $k$ the Boltzmann constant, $T$ the absorption temperature, $R_{s}$ the series resistance, and $q$ the electronic charge. By using (1), current $(I)$ can be plotted as a function of voltage $(V)$ as shown in the solid lines of Figure 3. From the fits, $R_{s}$ of the LED before and after depositing the luminescent Au nanoclusters was found to be 41 and $35 \Omega$, respectively, corresponding to an reduction in series resistance of $14.6 \%$. We suggest that the reduction of series resistance in the LEDs with nanoclusters is due to the effect of surface band bending. For the Au nanocrystals passivated by thiol ligands, the outer part (the ligand) of luminescent Au nanoclusters is negatively charged originated from the charge transfer from the outmost gold atoms to the surrounding adsorbed sulfur atoms [20]. The surrounding negative charge of $\mathrm{Au}$ nanoclusters can lead to a decrease of the built-in potential on the GaN surface for carriers flow to the metal [21]. Thus, the decrease of series resistance in LED could be due to the effect of the surface band bending. This effect also produces a change of EL energy, which will be discussed later.

Figure 4 shows the EL spectra of LEDs before (the open squares) and after (the solid line) the deposition of the luminescent $\mathrm{Au}$ nanoclusters onto the surface of LEDs. There is a main EL peak at around $2.82 \mathrm{eV}$ for the LEDs without deposition, corresponding to the emission from the InGaN wells. The EL peak slightly shifts toward higher energy region after

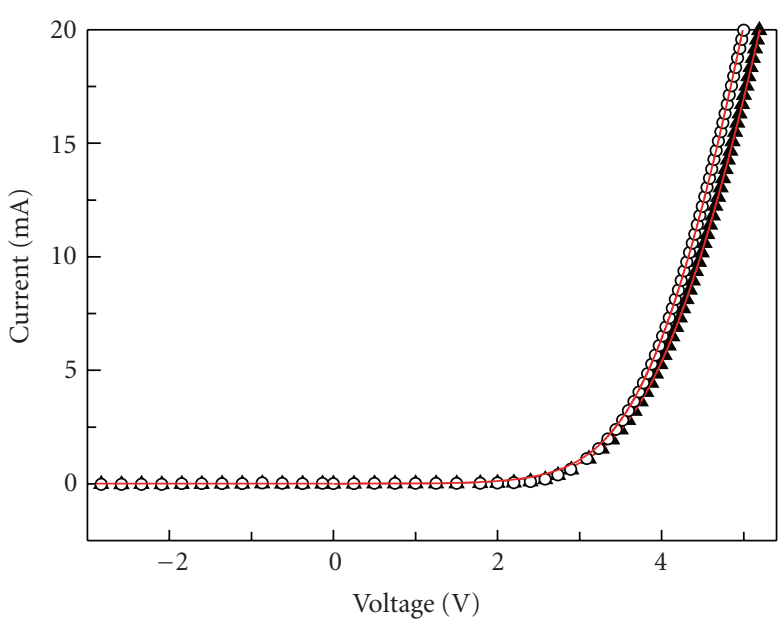

A Without deposition

○ With deposition

FIgURE 3: $I-V$ characteristics of the InGaN/GaN multiplequantum-well LEDs without (closed triangles) and with (open squares) deposition of the luminescent Au nanoclusters. The solid lines display the calculated $I$ using (1).

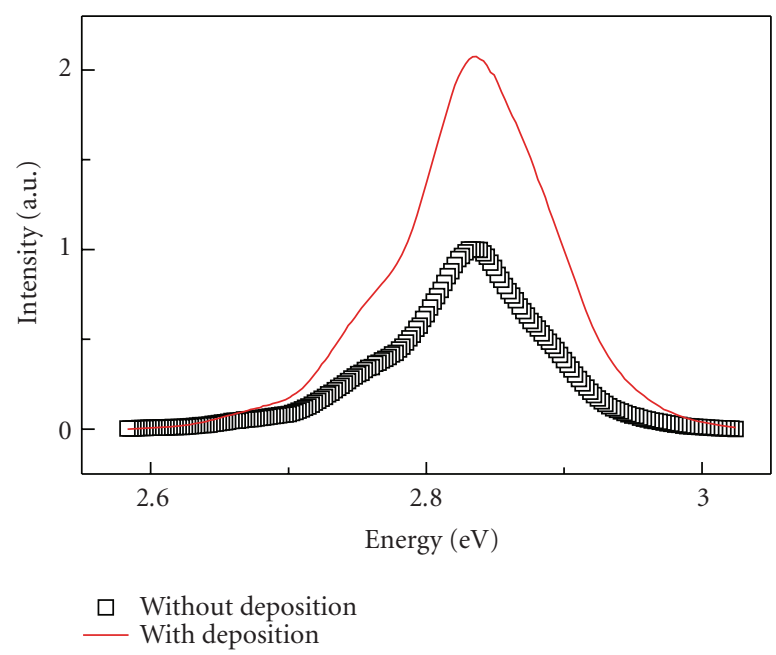

FIGURE 4: Electroluminescence spectra of InGaN/GaN multiplequantum-well LEDs without (open squares) and with (solid line) deposition of the luminescent Au nanoclusters.

deposition of the luminescent Au nanoclusters. The blue shift of EL can be explained by the effect of surface band bending mentioned above. For wurtzite nitride semiconductors, the strong polarization fields produce the built-in electrostatic fields parallel to the polar $\mathrm{c}$ axis of [0001] oriented $(\mathrm{Al}$, $\mathrm{Ga}$, In)N heterostructures. These electrostatic fields tend to bend the energy band structure of quantum wells, resulting in a redshift of transition energies (quantum-confined Stark effect) [22]. It has been reported that Au nanoparticles have a great influence on the surface potential of p-GaN [21]. We suggest that the luminescent Au nanoclusters reduce the surface band bending, leading to a decrease of the builtin electrostatic fields and a blueshift of EL peak, as shown 
in Figure 4. The EL intensity from LEDs with deposition of clusters shows more than two times enhancement compared to that from LEDs without the luminescent Au nanoclusters. The above results (the decrease in series resistance and the enhanced EL intensity) demonstrate that performance of the LEDs can be effectively improved by introduction of the luminescent Au nanoclusters.

Recently, the enhancement of the luminescence intensity for incorporation of metal nanoparticles has been related to a coupling of light to metal nanoparticles, caused by local plasmon excitation and interband transitions in the metal nanoparticles [3-5]. We suggest that the EL enhancement in Figure 4 can be interpreted by the increased scattering cross-section for light exciting Au nanoclusters, leading to the enhanced outcoupling of light from LEDs. For small particles with diameters well below the wavelength of light, the radiative scattering efficiency can be estimated from the absorption and scattering cross-sections $C_{\text {abs }}$ and $C_{\text {rad }}$ of the individual particles [23]:

$$
C_{\mathrm{sca}}=\frac{1}{2 \pi}\left(\frac{\omega}{c}\right)^{4}|\alpha|^{2}, \quad C_{\mathrm{abs}}=\frac{\omega}{c} \operatorname{Im}[\alpha],
$$

where $\alpha$ is the polarizability of the particle, determined by

$$
\alpha=3 V\left[\frac{\varepsilon(\omega) / \varepsilon_{m}-1}{\varepsilon(\omega) / \varepsilon_{m}+2}\right] \text {, }
$$

for a small spherical particle in vacuum, where $V$ is the the particle volume. $\varepsilon(\omega)$ is the dielectric function of the metal nanoclusters. $\varepsilon_{m}$ is the dielectric function of the embedding medium. The radiative scattering efficiency $Q_{\text {rad }}$, given by [23]

$$
Q_{\mathrm{rad}}=\frac{C_{\mathrm{sca}}}{C_{\mathrm{sca}}+C_{\mathrm{abs}}}
$$

represents the fraction of the extincted energy that is reradiated. According to the above Mie scattering theory, $Q_{\text {rad }}$ was obtained to be 2.2 , which agrees well with the EL enhancement $(\sim 2.1)$ in Figure 4 . The EL enhancement can thus be interpreted by the increased light scattering from $\mathrm{Au}$ nanoclusters.

Figure 5 displays the photovoltaic $I-V$ characteristics of the GaAs single junction solar cells were measured under AM 1.5 solar illumination at an intensity of $100 \mathrm{~mW} / \mathrm{cm}^{2}$ with a WACOM (WXS-130S-L2+) solar simulator. The dashed (solid) line represents the data without (with) deposition of the luminescent Au nanoclusters. The opencircuit voltage $\left(V_{\mathrm{OC}}\right)$ of $\mathrm{GaAs}$ solar cells was almost identical after depositing the luminescent Au nanoclusters. On the other hand, the incorporation of the Au nanoclusters yields a $14 \%$ increase in short-circuit current $\left(I_{\mathrm{SC}}\right)$ for the GaAs solar cells. This observation indicates that the absorption of the incident light in the active layers is enhanced, resulting in an increased photocurrent. The introduction of the luminescent $\mathrm{Au}$ nanoclusters may produce an increase of light scattering into the active layers in the solar cells, leading to the absorption enhancement of the incident light [16].

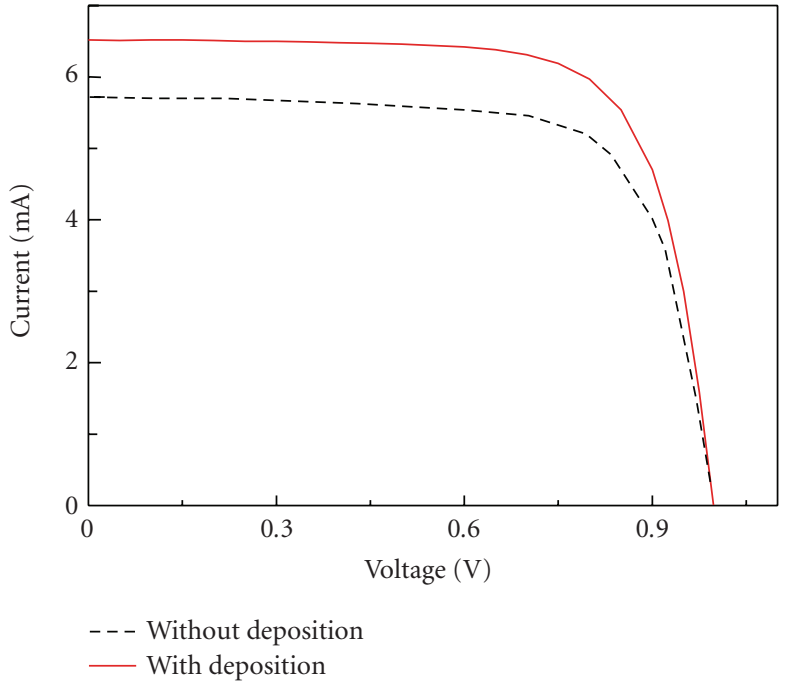

FIgure 5: Photovoltaic $I-V$ characteristics of the single-junction GaAs solar cells without (dashed line) and with (solid line) deposition of the luminescent Au nanoclusters under AM 1.5 solar illumination.

TABle 1: Photovoltaic $I-V$ results of the GaAs single-junction solar cells without and with incorporation of the luminescent $\mathrm{Au}$ nanoclusters. $V_{O C}$ is the open-circuit voltage, $I_{S C}$ is the short-circuit current, $F F$ is fill factor, and $\eta$ is conversion efficiency.

\begin{tabular}{lcccc}
\hline Sample & $V_{\text {oc }}(\mathrm{V})$ & $I_{\text {sc }}(\mathrm{mA})$ & $F F$ & $\eta(\%)$ \\
\hline Untreated & 0.99 & 5.72 & 0.73 & 16.2 \\
With Au nanoclusters & 0.99 & 6.52 & 0.74 & 18.7 \\
\hline
\end{tabular}

Conversion efficiency $\eta$ of solar cells from Figure 5 was calculated according to the following equation:

$$
\eta=V_{\mathrm{OC}} \times I_{\mathrm{SC}} \times \frac{F F}{P},
$$

where $P$ is the incident light intensity, and fill factor $(F F)$ is calculated by using the following equation:

$$
F F=\frac{(V \times I)_{\max }}{\left(V_{\mathrm{OC}} \times I\right)}
$$

where $I$ is the photocurrent. The obtained device parameters for the GaAs solar cell without (with) the luminescent $\mathrm{Au}$ nanoclusters on the cell surface are shown in Table 1. The incorporation of the luminescent $\mathrm{Au}$ nanoclusters yields a $13.9 \%$ (from 5.72 to $6.52 \mathrm{~mA}$ ) increase in shortcircuit current for the GaAs solar cells. From this result, the increase of $I_{\mathrm{SC}}$ leads to a $15.4 \%$ increase in energy conversion efficiency ( $\eta$ from 16.2 to $18.7 \%$ ) for the GaAs solar cells. To our knowledge, an increase of $15.4 \%$ in energy conversion efficiency is better than the previous reports on similar increase in energy conversion efficiency by using the large-size metallic nanoparticles $[6,7]$. The increase of conversion efficiency can also be explained by the effect of light scattering from nanoclusters on GaAs surface. We deduce that the absorption of the incident light in the 
absorbing layers of solar cells is enhanced due to enhanced light scattering by the luminescent $\mathrm{Au}$ nanoclusters. The observed increase in energy conversion efficiency in solar cells is thus caused by the enhanced light absorption in the absorbing layer.

\section{Summary}

The current-voltage characteristics and EL of the InGaN/ GaN MQW LEDs with the luminescent Au nanoclusters on the LED surface were investigated. The EL intensity and slope of the $I-V$ curve were found to increase after incorporation of the luminescent $\mathrm{Au}$ nanoclusters. In addition, a 13.9\% increase in short-circuit current and a $15.4 \%$ increase in energy conversion efficiency (from 16.2 to $18.7 \%$ ) are obtained for the GaAs solar cells where the luminescent Au nanoclusters have been incorporated. We suggest that the increase of EL intensity EL of the InGaN/GaN MQW LEDs and improvement of energy conversion efficiency in the In GaP solar cells are due to the increased radiative scattering by introducing the luminescent Au nanoclusters.

\section{Acknowledgments}

This project was supported in part by the National Science Council under Grant nos. NSC97-2112-M-033-004-MY3, and NSC 97-2627-B-033-002 and by the Institute of Nuclear Energy Research under Grant no. 962001INER0041.

\section{References}

[1] S. Franzen, J. C. W. Folmer, W. R. Glomm, and R. O'Neal, "Optical properties of dye molecules adsorbed on single gold and silver nanoparticles," The Journal of Physical Chemistry A, vol. 106, no. 28, pp. 6533-6540, 2002.

[2] M. Scolari, A. Mews, N. Fu, et al., "Surface enhanced Raman scattering of carbon nanotubes decorated by individual fluorescent gold particles," The Journal of Physical Chemistry C, vol. 112, no. 2, pp. 391-396, 2008.

[3] M. A. Mastro, J. A. Freitas Jr., O. Glembocki, et al., "Plasmonically enhanced emission from a group-III nitride nanowire emitter," Nanotechnology, vol. 18, no. 26, Article ID 265401, 4 pages, 2007.

[4] D.-M. Yeh, C.-F. Huang, C.-Y. Chen, Y.-C. Lu, and C. C. Yang, "Surface plasmon coupling effect in an InGaNGaN singlequantum-well light-emitting diode," Applied Physics Letters, vol. 91, no. 17, Article ID 171103, 3 pages, 2007.

[5] K. R. Catchpole and S. Pillai, "Surface plasmons for enhanced silicon light-emitting diodes and solar cells," Journal of Luminescence, vol. 121, pp. 315-318, 2006.

[6] D. Derkacs, S. H. Lim, P. Matheu, W. Mar, and E. T. Yu, "Improved performance of amorphous silicon solar cells via scattering from surface plasmon polaritons in nearby metallic nanoparticles," Applied Physics Letters, vol. 89, no. 9, Article ID 093103, 3 pages, 2006.

[7] B. P. Rand, P. Peumans, and S. R. Forrest, "Long-range absorption enhancement in organic tandem thin-film solar cells containing silver nanoclusters," Journal of Applied Physics, vol. 96, no. 12, pp. 7519-7526, 2004.

[8] O. Stenzel, A. Stendal, K. Voigtsberger, and C. von Borczyskowski, "Enhancement of the photovoltaic conversion efficiency of copper phthalocyanine thin film devices by incorporation of metal clusters," Solar Energy Materials and Solar Cells, vol. 37, no. 3-4, pp. 337-348, 1995.

[9] D. M. Schaadt, B. Feng, and E. T. Yu, "Enhanced semiconductor optical absorption via surface plasmon excitation in metal nanoparticles," Applied Physics Letters, vol. 86, no. 6, Article ID 063106, pp. 1-3, 2005.

[10] J. Zheng, C. Zhang, and R. M. Dickson, "Highly fluorescent, water-soluble, size-tunable gold quantum dots," Physical Review Letters, vol. 93, no. 7, Article ID 077402, 4 pages, 2004.

[11] Y. Negishi, K. Nobusada, and T. Tsukuda, "Glutathioneprotected gold clusters revisited: bridging the gap between gold(I)-thiolate complexes and thiolate-protected gold nanocrystals," Journal of the American Chemical Society, vol. 127, no. 14, pp. 5261-5270, 2005.

[12] G. C. Lica, B. S. Zelakiewicz, M. Constantinescu, and Y. Tong, "Charge dependence of surface plasma resonance on $2 \mathrm{~nm}$ octanethiol-protected $\mathrm{Au}$ nanoparticles: evidence of a freeelectron system," The Journal of Physical Chemistry B, vol. 108, no. 52, pp. 19896-19900, 2004.

[13] T. K. Sham, P. S. G. Kim, and P. Zhang, "Electronic structure of molecular-capped gold nanoparticles from X-ray spectroscopy studies: implications for coulomb blockade, luminescence and non-Fermi behavior," Solid State Communications, vol. 138, no. 10-11, pp. 553-557, 2006.

[14] L. L. Zhao, L. Jensen, and G. C. Schatz, "Surface-enhanced raman scattering of pyrazine at the junction between two Ag20 nanoclusters," Nano Letters, vol. 6, no. 6, pp. 1229-1234, 2006.

[15] E. Trave, G. Mattei, P. Mazzoldi, et al., "Sub-nanometric metallic Au clusters as efficient $\mathrm{Er}^{3+}$ sensitizers in silica," Applied Physics Letters, vol. 89, no. 15, Article ID 151121, 3 pages, 2006.

[16] M. D. Yang, Y. K. Liu, J. L. Shen, et al., "Improvement of conversion efficiency for multi-junction solar cells by incorporation of Au nanoclusters," Optics Express, vol. 16, no. 20, pp. 15754-15758, 2008.

[17] N. R. Jana and X. Peng, "Single-phase and gram-scale routes toward nearly monodisperse Au and other noble metal nanocrystals," Journal of the American Chemical Society, vol. 125, no. 47, pp. 14280-14281, 2003.

[18] S. Link, A. Beeby, S. FitzGerald, et al., "Visible to infrared luminescence from a 28-atom gold cluster," The Journal of Physical Chemistry B, vol. 106, no. 13, pp. 3410-3415, 2002.

[19] D. Lee, R. L. Donkers, G. Wang, A. S. Harper, and R. W. Murray, "Electrochemistry and Optical Absorbance and Luminescence of Molecule-like Au38 Nanoparticles," Journal of the American Chemical Society, vol. 126, no. 19, pp. 61936199, 2004.

[20] H. Häkkinen, R. N. Barnett, and U. Landman, "Electronic structure of passivated $\mathrm{Au}_{38}\left(\mathrm{SCH}_{3}\right)_{24}$ nanocrystal," Physical Review Letters, vol. 82, no. 16, pp. 3264-3267, 1999.

[21] H. K. Kim, H. W. Jang, and J.-L. Lee, "Photoemissioninduced charging of self-assembled Au nanoparticles on GaN substrates and the effect on surface band bending," Journal of Applied Physics, vol. 98, no. 10, Article ID 104309, 4 pages, 2005.

[22] E. Kuokstis, C. Q. Chen, M. E. Gaevski, et al., "Polarization effects in photoluminescence of C- and M-plane GaN/AlGaN multiple quantum wells," Applied Physics Letters, vol. 81, no. 22, pp. 4130-4132, 2002.

[23] H. R. Stuart and D. G. Hall, "Island size effects in nanoparticleenhanced photodetectors," Applied Physics Letters, vol. 73, no. 26, pp. 3815-3817, 1998. 

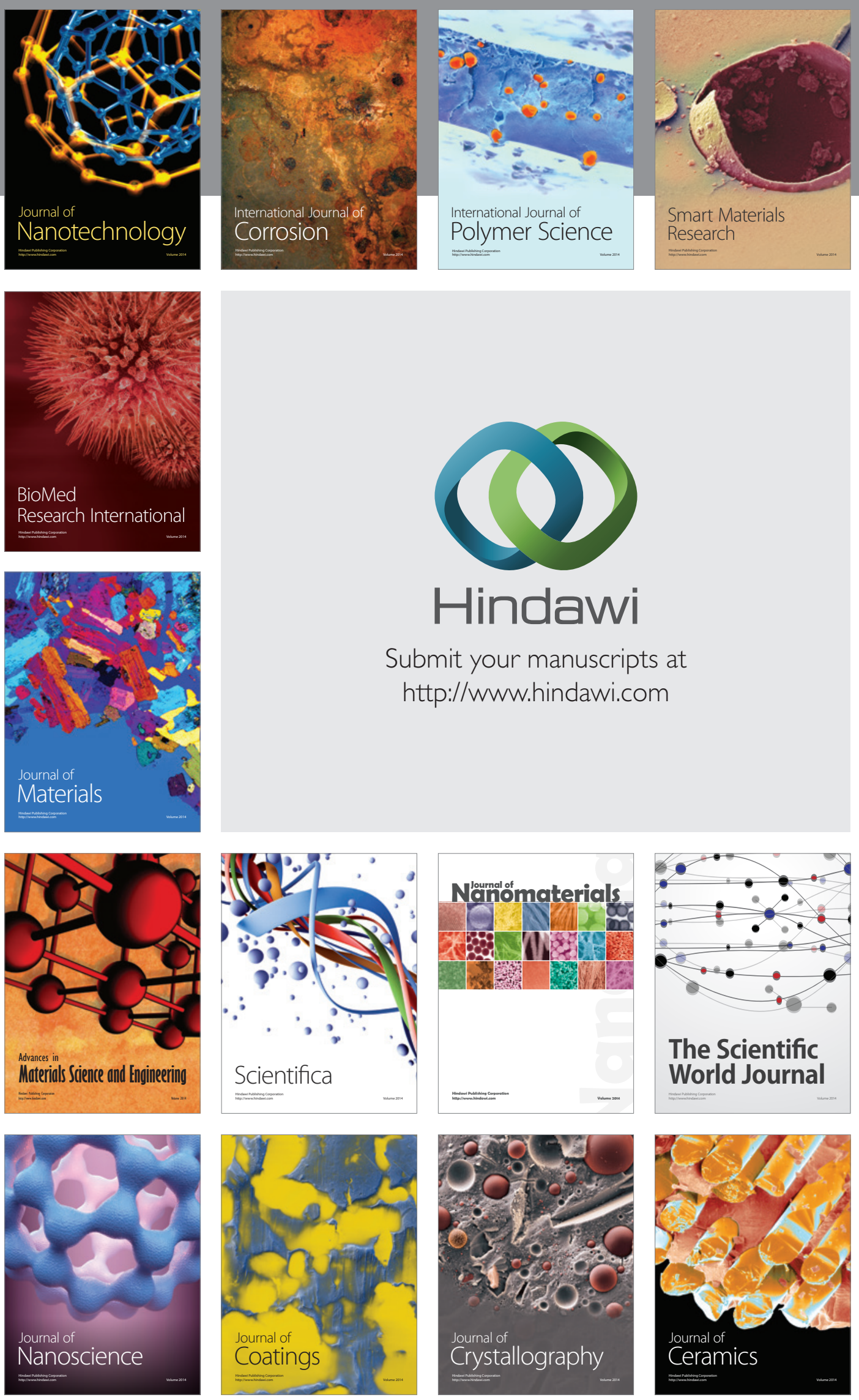

The Scientific World Journal

Submit your manuscripts at

http://www.hindawi.com

\section{World Journal}

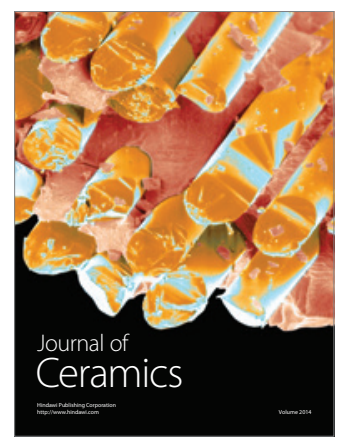

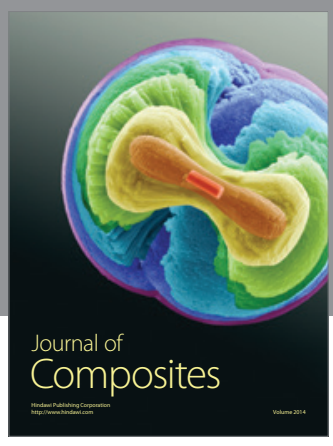
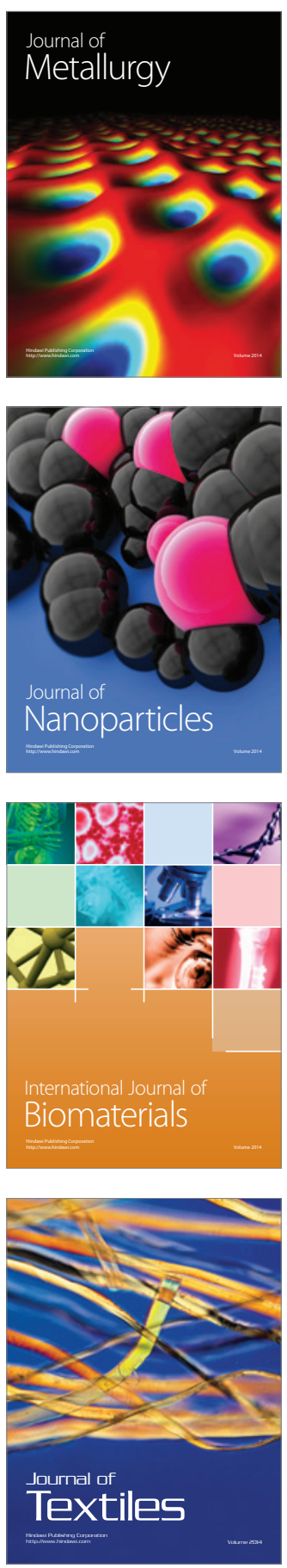